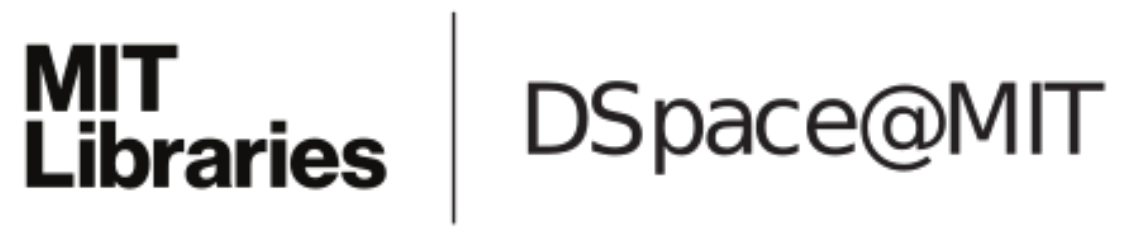

\author{
MIT Open Access Articles
}

Direct and quantitative broadband absorptance spectroscopy on small objects using Fourier transform infrared spectrometer and bilayer cantilever probes

The MIT Faculty has made this article openly available. Please share how this access benefits you. Your story matters.

Citation: Hsu, Wei-Chun et al. "Direct and Quantitative Broadband Absorptance Spectroscopy on Small Objects Using Fourier Transform Infrared Spectrometer and Bilayer Cantilever Probes." Applied Physics Letters 102.5 (2013): 051901. CrossRef. Web. (C) 2013 American Institute of Physics.

As Published: http://dx.doi.org/10.1063/1.4790184

Publisher: American Institute of Physics

Persistent URL: http://hdl.handle.net/1721.1/78314

Version: Final published version: final published article, as it appeared in a journal, conference proceedings, or other formally published context

Terms of Use: Article is made available in accordance with the publisher's policy and may be subject to US copyright law. Please refer to the publisher's site for terms of use. 


\section{AIP Appilied physics \\ Letters}

\section{Direct and quantitative broadband absorptance spectroscopy on small objects using Fourier transform infrared spectrometer and bilayer cantilever probes}

Wei-Chun Hsu, Jonathan K. Tong, Bolin Liao, Brian R. Burg, and Gang Chen

Citation: Appl. Phys. Lett. 102, 051901 (2013); doi: 10.1063/1.4790184

View online: http://dx.doi.org/10.1063/1.4790184

View Table of Contents: http://apl.aip.org/resource/1/APPLAB/v102/i5

Published by the American Institute of Physics.

Additional information on Appl. Phys. Lett.

Journal Homepage: http://apl.aip.org/

Journal Information: http://apl.aip.org/about/about_the_journal

Top downloads: http://apl.aip.org/features/most_downloaded

Information for Authors: http://apl.aip.org/authors

\section{ADVERTISEMENT}

\section{AIP Applied Physics Letters}

\section{EXPLORE WHAT'S NEW IN APL}

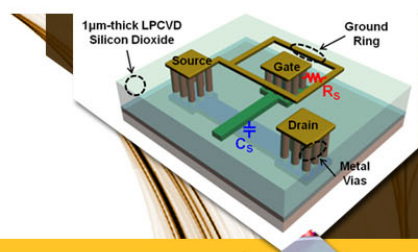

SURFACES AND INTERFACES

Focusing on physical, chemical, biological,

structural, optical, magnetic and electrical

properties of surfaces and interfaces, and more.. 


\title{
Direct and quantitative broadband absorptance spectroscopy on small objects using Fourier transform infrared spectrometer and bilayer cantilever probes
}

\author{
Wei-Chun Hsu, Jonathan K. Tong, Bolin Liao, Brian R. Burg, and Gang Chen ${ }^{\text {a) }}$ \\ Department of Mechanical Engineering, Massachusetts Institute of Technology, Cambridge, 02139 \\ Massachusetts, USA
}

(Received 27 December 2012; accepted 17 January 2013; published online 4 February 2013)

\begin{abstract}
A measurement platform is introduced that combines a bilayer cantilever probe with a Fourier transform infrared spectrometer to measure absolute spectral absorptance between wavelengths of $3 \mu \mathrm{m}$ and $18 \mu \mathrm{m}$ directly and quantitatively. The enhanced sensitivity provided by the cantilever probe enables the quantitative characterization of micro- and nanometer-sized samples. Validation of the technique is carried out by measuring the absorptance spectrum of a doped silicon thin film with a backside aluminum layer and found to agree well with the theoretical predictions. The presented technique is especially attractive for samples such as individual nanowires or nanoparticles, isolated molecules, powders, and photonic structures. (C) 2013 American Institute of Physics. [http://dx.doi.org/10.1063/1.4790184]
\end{abstract}

Light-matter interaction at the micro/nanoscale can exhibits a spectrum of exotic properties that may find important applications in various fields, such as effective light-trapping in photonic structures and larger-than-unity absorption cross-sections of particles and rods. ${ }^{1-3}$ In order to fully investigate these effects, a direct and quantitative technique for measuring the spectral absorptance, defined as the ratio of the absolute absorbed spectral power to the incident spectral power, of a micro/nano-sized sample is indispensable.

Fourier transform infrared (FTIR) spectroscopy is a well-established technique for measuring the optical properties of materials. Compared to dispersive spectrometers, FTIR has several important advantages, chief amongst which are the simultaneous measurement of all wavelengths in the illumination source during a scan ("multiplex advantage" or "Fellgett advantage") and the higher energy throughput which results in a higher signal-to-noise ratio ("throughput advantage" or "Jacquinot advantage"). ${ }^{4}$ By using different sample stages, a FTIR system can measure the transmittance and reflectance spectra of a sample. Traditionally, FTIR can only measure the absorptance spectra indirectly, by taking the difference of the transmittance and reflectance spectra. This approach inevitably introduces uncertainties and errors, since the portion of the diffusively scattered light cannot be measured easily. For bulk materials, indirect methods are usually adequate when making with proper provisions, such as the use of an integrating sphere and polishing. However, for small samples on the micro or nanometer scale, the compensative schemes are generally not available, and thus, the light scattered by the sample can cause significant errors.

Direct measurements of the spectral absorption at the micro/nano scale by FTIR have been previously demonstrated by using atomic force microscope (AFM) cantilever tips. $^{5-9}$ These techniques probed the temperature change, ${ }^{5,6}$ thermal expansion, ${ }^{7,8}$ or scattered field ${ }^{9}$ locally using cantile-

\footnotetext{
${ }^{\text {a) }}$ Author to whom correspondence should be addressed. Electronic mail: gchen2@mit.edu.
}

ver tips to extract absorption properties. In these studies, however, the measurements are qualitative in that the absolute absorbed power cannot be quantified due to the heat loss into the substrate. Furthermore, the use of a scanning probe tip allows local measurements only, whereas the optical response of isolated objects as a whole, such as suspended nanowires or nanoparticles, cannot be characterized. On the other hand, micro-fabricated tipless bilayer cantilevers (BCs) have been used as direct and quantitative thermal probes with an ultra-high power resolution $(\sim 4 \mathrm{pW}),{ }^{10-14}$ and at the same time, they can serve as holders for isolated samples. ${ }^{15,16}$ Bilayer cantilevers consist of two materials with different thermal expansion coefficients. When temperature changes, they bend due to the stress caused by the thermal expansion mismatch. These cantilevers have been used to measure the thermal conductance of thin films, ${ }^{17}$ near-field thermal radiation, ${ }^{18,19}$ the absorptance of a thin film of gold, ${ }^{20}$ and the thermal conductivity of polyethylene nanofibers. ${ }^{21}$ After light is absorbed by the sample, the energy will conduct as heat through the cantilever, leading to a temperature change that can be detected directly through the deflection of the cantilever. Since the sample is thermally isolated from the substrate, it is possible to quantify absolute absorbed power by appropriate calibrations. ${ }^{17-21}$

The tipless bilayer cantilever has been used to qualitatively study micro/nano-sized samples in the mid-infrared region using a monochromator ${ }^{22}$ and a FTIR spectrometer. ${ }^{23}$ In this paper, a quantitative measurement technique of the BC-FTIR system is introduced, which consists of a bilayer cantilever system merged with a FTIR system. The system combines the advantages inherent to both, the bilayer cantilever sensor and FTIR system. With appropriate calibrations, the BC-FTIR system can directly and quantitatively measure the absorptance of micro- and nanometer sized samples.

The BC-FTIR system comprises a light source assembly and a probe and detector assembly. The light source assembly includes a broadband light source (BLS), an interferometer (PerkinElmer, Spectrum GX), an optical coupler (OC) (JT 
Ingram Technologies, Fibermate $2^{\mathrm{TM}}$ FTIR Fiber Coupler), and a polycrystalline infra-red (PIR) fiber (ART Photonics). The probe and detector assembly includes the sample thermally coupled to the cantilever, a bilayer cantilever probe, a position sensitive detector (PSD) (On-Trak Photonics, PSM2$4 \mathrm{Q}$ ), and a computer to carry out Fourier transform analysis. Figure 1(a) shows specific components used in the BC-FTIR system. The spectral range is between $3 \mu \mathrm{m}$ and $18 \mu \mathrm{m}$ $\left(3333 \mathrm{~cm}^{-1}-555 \mathrm{~cm}^{-1}\right)$, dictated by the light source, the transmittance of the optical coupler, and the optical fiber.

The moving mirror in the Michelson interferometer of a FTIR system modulates the intensity of the broadband light source to a specific range of frequencies, providing a means of spectrally resolving electromagnetic radiation in the terahertz regime. The interference pattern is called an interferogram (Fig. 1(b)), and it can be decomposed into its spectral components via Fourier analysis. Each wavelength of light is modulated with a different temporal frequency, $f$, following the relation: ${ }^{4}$

$$
f=\frac{2 v}{\lambda}=2 v \eta
$$

where $v$ is the velocity of the movable mirror $(v=0.03 \mathrm{~cm} / \mathrm{s}$ in this paper), $\lambda$ is the wavelength, and $\eta$ is the wavenumber of the light source.

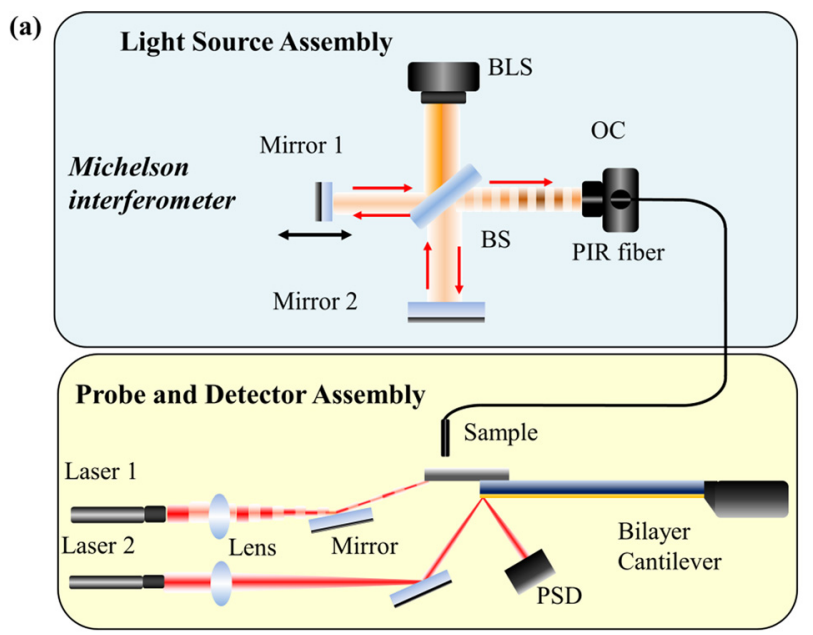

(b)
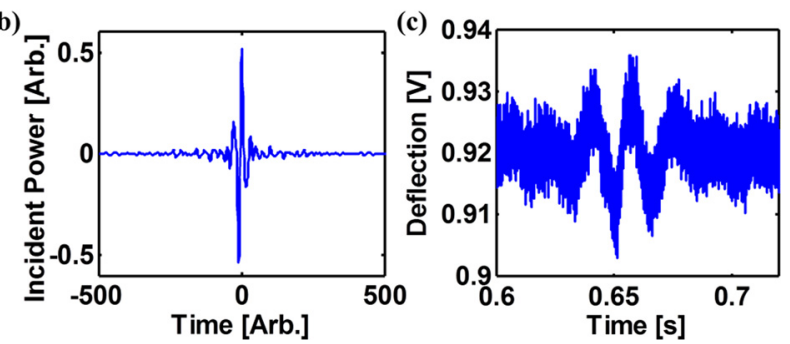

FIG. 1. Schematic of the BC-FTIR system. (a) It consists of a light source assembly and a probe and detector assembly. The light source assembly combines a BLS, a Michelson interferometer including a fixed mirror 1, a movable mirror 2, a beam splitter (BS), an OC, and a PIR fiber. The modulated light is coupled into a PIR fiber using an optical coupler. The probe and detector assembly are made up of the sample placed on a bilayer cantilever probe, which simultaneously acts as the sample stage and heat flux probe. The deflection of the cantilever is measured by a PSD and the signal is post-processed using Fourier transform analysis. (b) The incident power of an interferogram-pattern from the PIR fiber. (c) The absorbed signal from the bilayer cantilever. The PSD records the bilayer cantilever deflection signal in the expected interferogram pattern (mirror velocity, $v=0.03 \mathrm{~cm} / \mathrm{s}$ ).
The bilayer cantilever simultaneously functions as both the thermal probe and the sample holder in this system. The cantilever deflects when the sample absorbs light and heats up. The deflection of the cantilever is measured optically using a laser reflected off the cantilever onto a PSD. In our system, the cantilever vibrates in response to the modulated intensity of the incident light from the FTIR and the vibrational pattern is recorded in real time by the PSD. Data are then read by a data acquisition device (National Instruments, USB-6210) and analyzed using a Fourier transform (Fig. 1(c)). Compared to steady-state deflection, ${ }^{15-21}$ the dynamic behavior of bilayer cantilevers offers broadband information on the absorptive properties of samples in a single scan.

The spectral absorptance, $\alpha(\lambda)$, is quantitatively defined as

$$
\alpha(\lambda)=\frac{A(\lambda)}{I(\lambda)},
$$

where $A(\lambda)$ is the spectral absorbed power, and $I(\lambda)$ is the spectral incident power. To quantify both the absorbed and incident power, calibrations are needed, and they will be discussed in the next paragraph. To validate the BC-FTIR system, the absorptance of a silicon ( $\mathrm{Si}$ ) thin film with a backside aluminum ( $\mathrm{Al}$ ) layer is measured. In this case, the $\mathrm{Si} / \mathrm{Al}$ bilayer sample also serves as the bilayer cantilever probe. To this end, a $250 \mathrm{~nm} \mathrm{Al} \mathrm{film} \mathrm{is} \mathrm{sputtered} \mathrm{onto} \mathrm{a} \mathrm{Si}$ cantilever (Nanoworld, ARROW-TL1-50, $500 \mu \mathrm{m}$ length, $100 \mu \mathrm{m}$ width, and $0.5-2.5 \mu \mathrm{m}$ thickness) using a physical vapor deposition (PVD) process. The infrared light is incident on the Si because of the high reflectance of $\mathrm{Al}$ in the probed wavelength range.

For quantitative spectral measurements, four calibrations are required: the background spectrum calibration and the beam intensity distribution calibration to obtain the absolute incident power spectrum, $I(\lambda)$, and the frequency response calibration and power calibration for the absolute absorbed power spectrum, $A(\lambda)$.

First, the background spectrum calibration eliminates the impact of variations in the spectral intensity of the light source as well as atmospheric absorption. The incident power spectrum, $E_{f}(\eta)$, from the PIR fiber is measured by the FTIR system with a wavenumber resolution is $8 \mathrm{~cm}^{-1}$ (Fig. 2(a)). In this particular case, this spectrum is the Fourier transform of the interferogram in Fig. 1(b). Since a FTIR spectrometer only provides information of relative intensity on each spectral component, a thermopile power meter (Newport, High Sensitivity Thermopile Sensor, 818P-001-12) is used to measure the total power to properly normalize the incident spectrum. Since the power level leaving the PIR fiber is so small that it is susceptible to environmental noise, it is not directly measured by the thermopile. Instead, it is extrapolated from both the total power and the background spectrum, $E_{B}(\eta)$ (measured by the thermopile and FTIR detector, respectively) coming out of the FTIR system (before entering the PIR fiber) with different aperture sizes controlled by an iris called Bstop, while assuming that the absolute power is proportional to the area enclosed by the power spectrum curves ("spectral area") (Fig. 2(b)). The absolute power coming out of the PIR fiber is estimated through $P_{\text {fiber }}=P_{B-\text { stop }} \times\left(A_{\text {fiber }} / A_{B-\text { stop }}\right)$, 

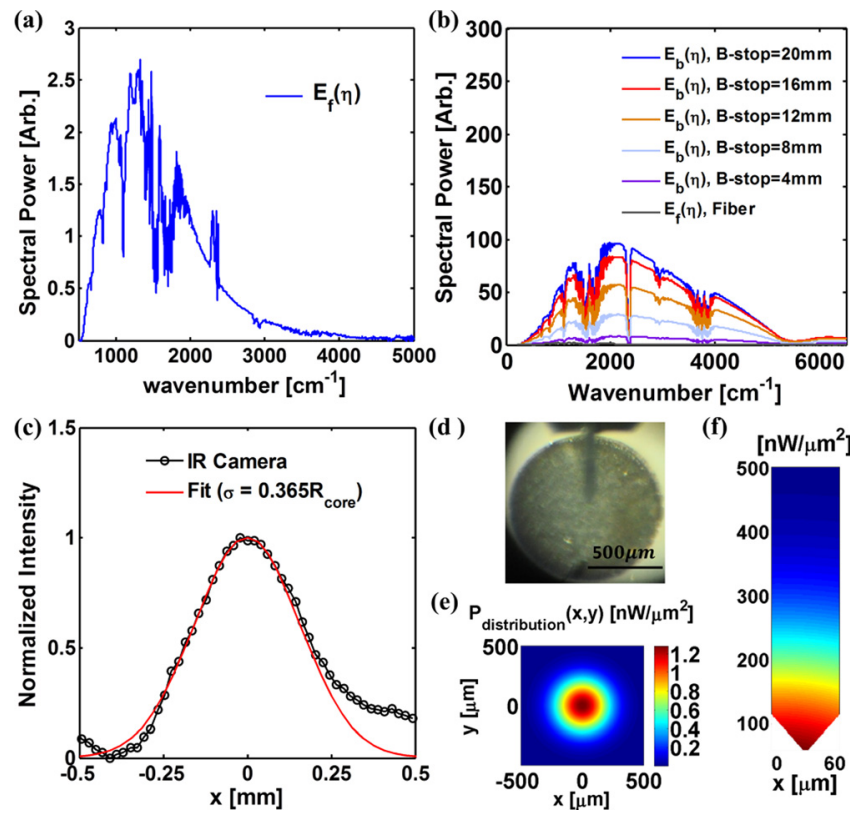

FIG. 2. Background and intensity distribution calibrations of BC-FTIR system. (a) The incident power spectrum on the cantilever (background spectrum exiting from the PIR fiber), $E_{f}(\eta)$. The spectrum is calculated by taking the Fourier transform of the interferogram in Fig. 1(b) obtained through the FTIR system. (b) The background spectrum calibration. $E_{B}(\eta)$ is the power spectrum leaving the FTIR system (before entering the PIR fiber) with different aperture sizes controlled by an iris called B-stop. (c) Beam intensity distribution leaving the PIR fiber, measured by an IR camera and fitted to a Gaussian profile with $\sigma=0.365 R_{\text {core }}$. (d) Top view of the bilayer cantilever, covering only a portion of the light coming out of the PIR fiber. (e) The intensity distribution from PIR fiber. (f) The incident intensity distribution on the bilayer cantilever. $P_{\text {incident }}$ is the spatial integral of the intensity on the cantilever.

where $P_{B-\text { stop }}$ and $A_{B-\text { stop }}=\int E_{B}(\eta) d \eta$ are the total power and the spectral areas with different B-stop sizes entering the PIR fiber, respectively. $A_{f i b e r}=\int E_{f}(\eta) d \eta$ is the spectral area leaving the PIR fiber, where $E_{f}(\eta)$ is the incident power spectrum on the cantilever (background spectrum exiting from the PIR fiber). The average extracted power from the PIR fiber is $P_{\text {fiber }}=197.3 \pm 6.5 \mu \mathrm{W} .^{24}$

Second, the beam intensity distribution calibration considers the non-uniform spatial profile of the output intensity from the PIR fiber. Since the diameter of the PIR fiber is larger than the dimensions of the cantilever, only a fraction of the output from the fiber will be intercepted. In order to evaluate the incident power on the sample accurately, the intensity distribution and the alignment of the sample to the PIR fiber must be known with a high degree of precision. To illustrate this point, the beam intensity profile is measured using an IR camera (FLIR system, ExaminIR ${ }^{\mathrm{TM}}$ ) and fitted with a Gaussian function normalized to $P_{\text {fiber }}$ from the background spectrum calibration. ${ }^{25}$ Two of these curves are shown in Fig. 2(c), and the best fitting parameter using the least squares method is $\sigma=0.365 R_{\text {core }}$, where $R_{\text {core }}=430 \pm 10 \mu \mathrm{m}$ is the core radius of the PIR fiber, and $\sigma$ is the standard deviation. Based on the alignment of the PIR fiber and the sample shown in Fig. 2(d)), a spatial two-dimensional (2D) intensity distribution, $P_{\text {distribution }}(x, y)$ (Fig. 2(e)), mapped by the Gaussian fitting curve, is then used to account for the incident power on the cantilever. This incident power on the cantilever is calculated through $P_{\text {incident }}=\iint_{A_{\text {cantilever }}} P_{\text {distribution }}(x, y) d x d y$, where
$A_{\text {cantilever }}$ is the spatial cross-sectional area of the cantilever (Fig. 2(f)). From the intensity distribution calibration, the incident power on the cantilever is $P_{\text {incident }}=10.5 \pm 2.5 \mu \mathrm{W}$, and it is used to normalize the total spectral area in Fig. 2(a): $I(\lambda)=E_{f}(\eta) \times\left[P_{\text {incident }} / \int E_{f}(\eta) d \eta\right] \times[d \eta / d \lambda]$, where the relation between $\lambda$ and $\eta$ is given by Eq. (1). The normalized incident power spectrum, $I(\lambda)$, is shown in Fig. 4(a).

For calculating the absorptance spectrum, $\alpha(\lambda)$, through Eq. (2), the raw absorbed power spectrum, $E_{a}(f)$ (Fig. 4(a)), recorded by PSD (with the sampling rate: 250000 samples per second and the total sampling time: $1 \mathrm{~s}$ ) required two more calibrations to be quantified to the absolute absorbed power spectrum, $A(\lambda)$. The third calibration, the frequency response calibration, corrects the phase lag of the periodic heat input flowing through the cantilever. This phase lag, which is related to the thermal time constant of the cantilever, causes a reduction of the vibrational amplitude with increasing input modulation frequency. To characterize the frequency response of the cantilever, an additional modulated laser (Lasermate Group, LTC6704A5-T) is used to periodically heat the cantilever from the $\mathrm{Al}$ side at a $50 \%$ duty cycle (laser 1 in Fig. 1(a)). The vibrational amplitude, $y(f)$, of the $\mathrm{Si} / \mathrm{Al}$ bilayer cantilever is measured in air through the PSD at different modulation frequencies (Fig. 4(b)). The frequency response curve is expressed as $F(f)=y(f) / P_{\text {absorbed }}$, where $P_{\text {absorbed }}$ is the absorbed power. A peak in the frequency response is observed around $4550 \mathrm{~Hz}$, corresponding to the resonance frequency of the cantilever. More importantly, considering the reduced response at high frequencies, the working region is chosen to be in the range of $30-1000 \mathrm{~Hz}$ to maintain a measurable signal while avoiding low frequency noise. When the vibrational amplitude of the absorbed power is kept constant, it can be quantified through an adequate power calibration.

Finally, the power calibration consists of three steps to quantify $P_{\text {absorbed }}$. First, the incident power on the cantilever
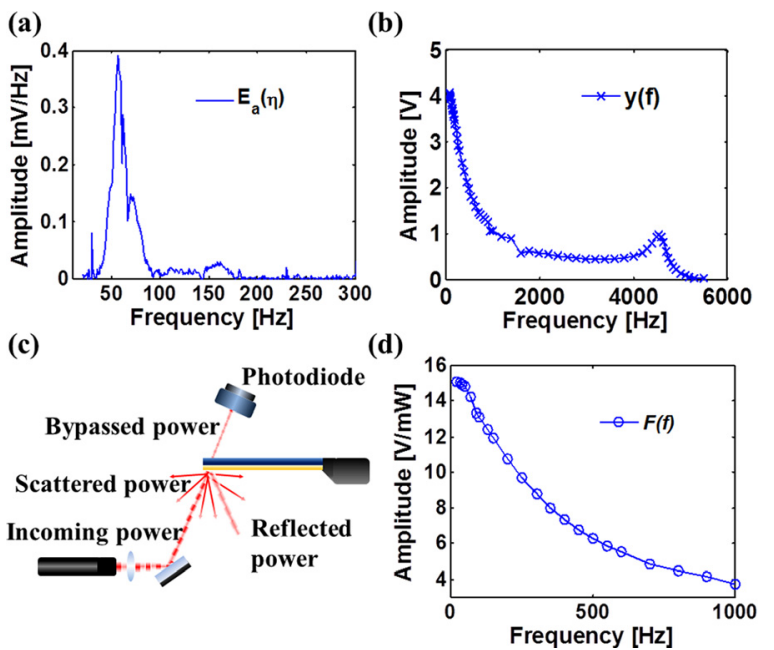

FIG. 3. Frequency response and power calibrations of the BC-FTIR system. (a) The raw absorbed power spectrum calculated from the Fourier transform of the interferogram in Fig. 1(c). (b) Experimental frequency response of the bilayer cantilever. (c) The schematic of the incoming, bypassed, reflected, and scattered power (d) Frequency response and power calibrations translate the vibrational amplitude on PSD to the absolute absorbed power of the cantilever. 
(a)

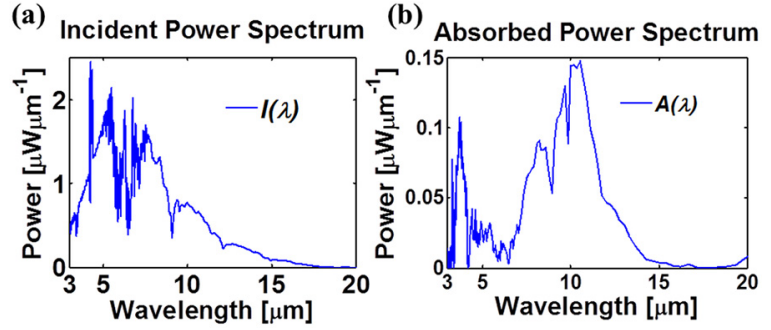

(c)

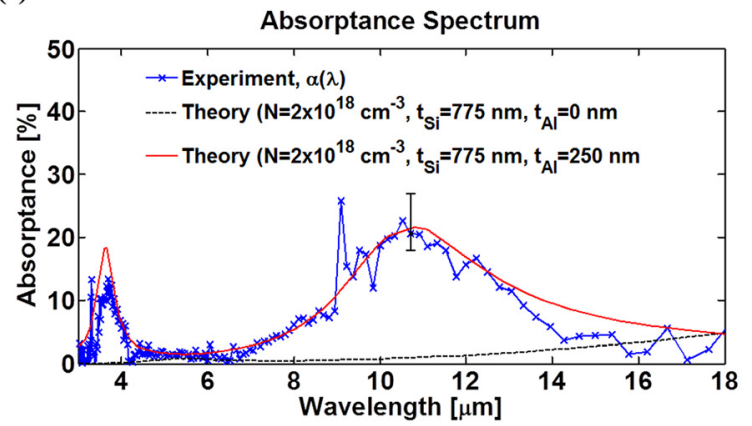

FIG. 4. Quantitative spectra of the Si/Al thin film. (a) The calibrated incident power spectrum. (b) The calibrated absorbed power spectrum. (c) The experimental and theoretical absorptance spectra. These spectra are measured with a mirror velocity of $0.03 \mathrm{~cm} / \mathrm{s}$ and averaged over 50 scans.

of the modulated laser is obtained by subtracting the bypassed power (not intercepted by the cantilever) from the incoming power (Fig. 4(c)). The bypassed power $(0.1089 \pm 0.001 \mathrm{~mW})$ and incoming power $(2.893 \pm 0.05 \mathrm{~mW})$ are measured using a photodiode (Newport, 818-UV). Second, the absorptance of the metal film, Al in this case, is measured using a conventional spectrometer (PerkinElmer, Lambda $950 \mathrm{UV} / \mathrm{Vis} / \mathrm{NIR}$ spectrophotometer) by depositing $\mathrm{Al}$ on a $\mathrm{Si}$ wafer using the same PVD process to coat the cantilever. The metal is optically thick so that substrate effects are negligible. The absorptance of the $250 \mathrm{~nm}$ aluminum film is $0.1074 \pm 0.0004$ at the laser wavelength of $670 \mathrm{~nm}$. After measuring the absorptance, the absorbed power $\left(P_{\text {absorbed }}=0.2990 \pm 0.0065 \mathrm{~mW}\right)$ on the cantilever is calculated by multiplying the absorptance with the incident power $(2.784 \pm 0.05 \mathrm{~mW})$. Note that the absorbed power is determined in this manner because the scattered light by the cantilever is difficult to measure. Third and finally, the deflection amplitude on the PSD then corresponds to the known absorbed power on the cantilever at each frequency. With the known frequency response and power calibration, the raw absorbed power spectrum, $E_{a}(f)$ (Fig. 4(a)), from the Fourier transform of the deflection signal in Fig. 1(c) is divided by the frequency response curve, $F(f)$, to determine the quantitative absorbed power spectrum, $A(\lambda)=\left[E_{a}(f) / F(f)\right] \times[d f / d \lambda]$, where the $f$ can be converted to $\lambda$ through Eq. (1) (Fig. 3(b)). Consequently, the quantitative absorptance spectrum, $\alpha(\lambda)$, is calculated through Eq. (2).

The transfer matrix method offers an estimation of the absorptance of a Si $(775 \mathrm{~nm}) / \mathrm{Al}(250 \mathrm{~nm})$ thin film, ${ }^{26,27}$ and the theory matches well with the quantitative averaged results of experiments presented in Fig. 3(c). The theoretical results use the n-type $\mathrm{Si}$ with a doping concentration of $2 \times 10^{18}\left[\mathrm{~cm}^{-3}\right]$. The uncertainty of thickness variation and resistance of the Si layer provided by Nanoworld are 500$2500 \mathrm{~nm}$ and $0.01-0.025 \Omega \mathrm{cm}$ (the corresponding concentra- tion is $\left.1 \times 10^{18} \sim 5 \times 10^{18} \mathrm{~cm}^{-3}\right),{ }^{28}$ and the fitting values of $775 \mathrm{~nm}$ and $2 \times 10^{18} \mathrm{~cm}^{-3}$ are within these ranges. ${ }^{24}$ Constructive interferences from the refracted and reflected electromagnetic waves in the Si layer are observed around $3.8 \mu \mathrm{m}$ and $10.5 \mu \mathrm{m}$. Compared to monolayer Si, the absorptance of a $\mathrm{Si} / \mathrm{Al}$ thin film is stronger in the range below $18 \mu \mathrm{m}$. Additionally, interference effects are not observable in a $\mathrm{Si}$ monolayer because of the weaker reflection at the $\mathrm{Si}$ / Air boundary. The maximum error of the absorptance spectrum occurs at $10.5 \mu \mathrm{m}$, and its corresponding upper and lower limits are indicated by the error bar.

The measurement platform presented in this paper allows for quantitative broadband absorptance spectra measurements on micro/nano-scaled samples. By combining a micro-fabricated bilayer cantilever with a FTIR system, the broadband absorptance spectrum of a small sample can be measured in a single scan. Compared to conventional dispersive spectrometers, this platform fundamentally allows for faster data acquisition and a higher signal-to-noise ratio. In addition, the use of a bilayer cantilever probe enables the direct and quantitative measurement of absorptance spectra. Experimental results presented so far are based on commercially available cantilevers. It should be emphasized that different variations of the cantilever-based sensor can further improve the sensitivity and accuracy of the system. A new structural design, which consists of a bi-arm cantilever platform that decouples the sample stage from the probe, was recently introduced. ${ }^{29}$

The authors thank Dr. S. Yerci and T. H. Huang for supports with the LabView program; A. Lenert for infrared imaging (Fig. 2(c)); Dr. S. Chen and W. C. Chen for the help with the derivation of the dynamic model of the bilayer cantilever; D. Kraemer and K. McEnaney for access to the vacuum chamber setup; J. Sullivan for absorptance measurements on the Al film; and J. Y. Kuan, K. H. Chu, G. Ni, P. Sambegoro, Professor A. Minnich, Dr. A. Muto, K. Collins, T. McClure, and A. Marconnet for helpful discussions. This work was funded by DOE BES Grant No. DE-FG0202ER45977 (W. C. Hsu and B. R. Burg), DOD MURI via UIUC FA9550-08-1-0407 (J. K. Tong, B. R. Burg, and G. Chen), and partial post-doc fellowship to B. R. Burg from the National Research Fund, Luxembourg (Grant No. 893874).

\footnotetext{
${ }^{1}$ A. Mavrokerfalos, S. E. Han, S. Yerci, M. S. Branham, and G. Chen, Nano Lett. 12(6), 2792-2796 (2012).

${ }^{2}$ S. E. Han and G. Chen, Nano Lett. 10(11), 4692-4696 (2010).

${ }^{3}$ L. Cao, J. S. White, J. S. Park, J. A. Schuller, B. M. Clemens, and M. L. Brongersma, Nature Mater. 8, 643-647 (2009).

${ }^{4} \mathrm{P}$. R. Griffiths and J. A. de Haseth, Fourier Transform Infrared Spectrometry (Wiley, New York, 2007).

${ }^{5}$ A. Hammiche, H. M. Pollock, M. Reading, M. Claybourn, P. H. Turner, and K. Jewkes, Appl. Spectrosc. 53(7), 810-815 (1999).

${ }^{6}$ A. Hammiche, L. Bozec, M. J. German, J. M. Chalmers, N. J. Everall, G. Poulter, M. Reading, D. B. Grandy, F. L. Martin, and H. M. Pollock, Spectroscopy 19(2), 20-42 (2004), available at http://www.spectroscopy online.com/spectroscopy/data/articlestandard//spectroscopy/132005/152794/ article.pdf.

${ }^{7}$ A. Dazzi, R. Prazeres, F. Glotin, and J. M. Ortega, Opt. Lett. 30(18), 2388-2390 (2005)

${ }^{8}$ C. Prater, K. Kjoller, D. Cook, R. Shetty, G. Meyers, C. Reinhardt, J. Felts, W. King, K. Vodopyanov, and A. Dazzi, Microsc. Anal. 24(3), 5-8 (2010),
} 
available at http://www.stanford.edu/ vodopyan/publications/2010_Microscopy_and_Analysis_Prater.pdf.

${ }^{9}$ F. Huth, A. Govyadinov, S. Amarie, W. Nuansing, F. Keilmann, and R. Hillenbrand, Nano Lett. 12(8), 3973-3978 (2012).

${ }^{10}$ J. R. Barnes, R. J. Stephenson, M. E. Welland, Ch. Gerber, and J. K. Gimzewski, Nature 372(3), 79-81 (1994).

${ }^{11}$ J. Lai, T. Perazzo, Z. Shi, and A. Majumdar, Sens. Actuators, A 58, 113 119 (1997).

${ }^{12}$ S. Sadat, Y. J. Chua, W. Lee, Y. Ganjeh, K. Kurabayashi, E. Meyhofer, and P. Reddy, Appl. Phys. Lett. 99, 043106 (2011).

${ }^{13}$ J. R. Barnes, R. J. Stephenson, C. N. Woodburn, S. J. O'Shea, M. E. Welland, T. Rayment, J. K. Gimzewski, and C. Gerber, Rev. Sci. Instrum. 65(12), 3793-3798 (1994).

${ }^{14}$ J. Varesi, J. Lai, T. Perazzo, Z. Shi, and A. Majumdar, Appl. Phys. Lett. 71(3), 306-308 (1997).

${ }^{15}$ E. T. Arakawa, N. V. Lavrik, and G. Datskos, Appl. Opt. 42(10), $1757-$ 1762 (2003).

${ }^{16}$ P. G. Datskos, S. Rajic, M. J. Sepaniak, N. Lavrik, C. A. Tipple, L. R. Senesac, and I. Datskou, J. Vac. Sci. Technol. B 19, 1173-1179 (2001).

${ }^{17}$ S. Shen, A. Narayanaswamy, S. Goh, and G. Chen, Appl. Phys. Lett. 92, 063509 (2008).

${ }^{18}$ S. Shen, A. Narayanaswamy, and G. Chen, Nano Lett. 9, 2909-2913 (2009).
${ }^{19}$ S. Shen, A. Mavrokefalos, P. Sambegoro, and G. Chen, Appl. Phys. Lett. 100, 233114 (2012).

${ }^{20}$ A. Narayanaswamy and N. Gu, J. Heat Transfer 133, 042401 (2011).

${ }^{21}$ S. Shen, A. Henry, J. Tong, R. Zheng, and G. Chen, Nat. Nanotechnol. 5, 251-255 (2010).

${ }^{22}$ B. Kwon, C. Wang, K. Park, R. Bhargava, and W. P. King, Nanoscale Microscale Thermophys. Eng. 15, 16-28 (2011).

${ }^{23}$ L. Tetard, A. Passian, R. H. Farahi, B. H. Davison, and T. Thundat, Opt. Lett. 36, 3251-3253 (2011).

${ }^{24}$ See supplementary material at http://dx.doi.org/10.1063/1.4790184 for more details on this extrapolation of the background spectrum calibration and the detailed discussion on the theoretical fitting of the absorptance of the Si/Al thin film.

${ }^{25}$ D. Marcuse, J. Opt. Soc. Am. 68(1), 103-109 (1978).

${ }^{26} \mathrm{G}$. Chen, Nanoscale Energy Transport and Conversion (Oxford University Press, New York, 2005).

${ }^{27}$ A. Wig, A. Passian, E. Arakawa, T. L. Ferrell, and T. Thundat, J. Appl. Phys. 95(3), 1162-1165 (2004).

${ }^{28}$ S. M. Sze and K. K. Ng, Physics of Semiconductor Devices (Wiley, New Jersey, 2007).

${ }^{29}$ B. R. Burg, J. K. Tong, W. C. Hsu, and G. Chen, Rev. Sci. Instrum. 83, 104902 (2012). 